\title{
Brain Imaging Study on the Pathogenesis of Depression \& Therapeutic Effect of Selective Serotonin Reuptake Inhibitors
}

\author{
Qi Meng ${ }^{1,2}$, Aixia Zhang ${ }^{1}$, Xiaohua Cao', Ning Sun ${ }^{1}$, Xinrong Li ${ }^{1}$, YunQiao Zhang ${ }^{1,2}$, and Yanfang Wang ${ }^{1 凶}$ \\ ${ }^{1}$ Department of Psychiatry, First Hospital of Shanxi Medical University, Taiyuan, China \\ ${ }^{2}$ Shanxi Medical University, Taiyuan, China
}

\begin{abstract}
Objective Predefining the most effective treatment for patients with depressive disorders remains a problem. We will examine the differential brain regions of gray matter (GM) in major depressive disorder (MDD) patients and the relationship between changes in their volume and the efficacy of early antidepressant treatment using magnetic resonance imaging (MRI).

Methods 159 never-medicated patients with first-episode MDD and 53 normal control subjects (NCs) were enrolled. The brains were scanned by MRI and measured with the 17-item Hamilton Depression Rating Scale (HAMD-17) at baseline and after 2 weeks of treatment with selective serotonin reuptake inhibitor (SSRI)s, and the non-responder group and responder group were obtained. The patients were analyzed by voxel-based morphological (VBM) and SPSS software. Receiver operator characteristics (ROC) analysis was performed for the difference between the responder group and the non-responder group in the differential brain regions, and Pearson correlations were computed between volume size and HAMD score reduction rate.

Results Smaller GM volume of the right superior temporal gyrus (STG), and the orbital parts of the right medial frontal gyrus and right inferior frontal gyrus were observed in MDD versus the NCs. The non-responder group demonstrated a significant volume reduction at the right STG compared with the responders, but no corresponding change in orbital part of right medial frontal gyrus and right inferior frontal gyrus. ROC analysis showed that Accuracy $=71.2 \%$. There was a positive correlation between the STG gray matter volume and the HAMD-17 score reduction rate $(\mathrm{r}=0.347, \mathrm{p}=0.002)$.

Conclusion The study results confirmed the local changes in brain structure in MDD and may initially predict the early treatment response produced by SSRIs as antidepressants.

Psychiatry Investig 2020;17(7):688-694
\end{abstract}

Key Words Major depressive disorder, Voxel-based morphometry, Gray matter, Predict efficacy.

\section{INTRODUCTION}

Major depressive disorder (MDD) is a chronic disease that affects the social and occupational function of patients. According to the World Health Organization, the number of people who suffer from depression is estimated to be 322 million worldwide, accounting for approximately $4.4 \%$ of the world population. ${ }^{1} \mathrm{MDD}$ is in a complex and heterogeneous manifestation characterized by negative moods, psychomotor disorders, and

Received: February 2, 2020 Revised: March 29, 2020

Accepted: May 18, 2020

$\triangle$ Correspondence: Yanfang Wang, MD

Department of Psychiatry, First Hospital of Shanxi Medical University, No 85 Jiefang Road, Taiyuan 030001, China

Tel: +861 393455 6065, E-mail: wangyanfang123868@163.com

(c) This is an Open Access article distributed under the terms of the Creative Commons Attribution Non-Commercial License (https://creativecommons.org/licenses/by$\mathrm{nc} / 4.0$ ) which permits unrestricted non-commercial use, distribution, and reproduction in any medium, provided the original work is properly cited. cognitive deficits. Due to its high prevalence and diverse symptoms, exploring the pathogenesis of MDD is essential for improving clinical diagnosis and treatment evaluation. ${ }^{2}$

Psychiatrists usually visit or observe patients to assess their mental symptoms for the diagnosis of MDD. As such, early identification of MDD is challenging without the establishment of visual diagnostic criteria. The main reason is that the underlying neurobiological mechanism of MDD remains unknown. To address this problem, neuroimaging techniques are widely used to investigate brain changes in MDD, and a large number of studies have also demonstrated that cortical volume reduction is usually region-specific, which can provide valuable additional information for characterizing the specificity of neuroanatomical changes related to this disease.

Voxel-based morphometry (VBM) is a technique used to analyze brain MR images on a voxel-by-voxel basis. It is an automated technique for the whole brain that allows a voxel-wise 
comparison of the local concentration and volume of gray matter between groups. In the past few decades, VBM has been widely used in MDD researches to identify neuroanatomical abnormalities related to this condition. ${ }^{3,4}$ In those reports concerning structural abnormalities, volume reduction in the frontal lobe region (especially anterior cingulate gyrus, orbitofrontal cortex, prefrontal cortex, and hippocampus) is most consistent. ${ }^{5,6}$ Multiple studies have shown that the fronto-limbic circuit is of critical significance for the pathogenesis of depression. ${ }^{7-9}$ Gray matter changes that have been identified in the fronto-limbic network for never-medicated MDD patients are closely related to the clinical symptoms including mood disorders, cognitive deficits and rumination. ${ }^{2}$ Guo et al. ${ }^{10,11}$ studied patients with acute onset and without medication, and found that the structural deficits of MDD may unidirectionally destroy the causal topology of the cortico-limbic-cerebellar circuit. At the same time, they also found that the GM volume in the parietal-temporal regions decreased, and the average GM volume value of the left angular gyrus was negatively correlated with the episode number and the duration of illness.

At present, there are many antidepressant options in clinical applications. However, there is no objective indicator for selecting effective medications, as clinicians often prescribe medications to the patients based on their experience. For MDD, selective serotonin reuptake inhibitors (SSRIs) are novel antidepressants in current treatment strategies as first-line treatment for patients with moderate to severe depression. As most frequently selected drugs for this condition, SSRIs had good efficacy and few adverse reactions. However, such antidepressants usually take effect after 2-4 weeks of administration coupled with unsatisfactory efficacy found in about $30-40 \%$ of patients. ${ }^{12,13}$ Delayed or ineffective antidepressant treatment can cause considerable losses to individuals and the economy. ${ }^{14}$ Previously untreated MDD can lead to increase of non-psychiatric medical expenses by 2 to 7 times. ${ }^{15,16}$ Therefore, it is crucial to select highly specific and effective antidepressant.

This was the first study to combine changes in gray matter volume affected by brain structure with early antidepressant treatment response. In this study, the never-medicated patients with first-episode MDD from outpatient settings were collected and treated with antidepressant SSRIs alone for 2 weeks, followed by response rate assessment as per HAMD-17, thereby investigating the relationship between brain structural changes in patients with depressive disorder and early clinical response. All these were done to consider whether brain imaging could be a potential approach to predicting early clinical efficacy in MDD patients.

\section{METHODS}

\section{Participants}

From September 2009 to December 2018, never-medicated patients with first-episode major depressive disorder (MDD) were recruited from the Outpatient Department of Psychiatry, First Hospital of Shanxi Medical University, as the study group. The MDD diagnosis was made using the Diagnostic and Statistical Manual of Mental Disorders-Fifth Edition (DSM-5). ${ }^{17}$ All the subjects were examined through a structured interview by the professionally trained postgraduate students from the Department of Psychiatry using the Chinese version of Mini International Neuropsychiatric Interview, and HAMD-17 were used to evaluate the severity of depression. Healthy subjects were recruited as a normal control (NC) group in the meantime. By excluding the non-eligible data, there were a total of 159 MDD patients and 53 NCs.

Inclusion criteria for study group: 1) Han Chinese descent, aged 18-55 years old, right-handed; 2) first-episode not treated with antidepressants or others, and receiving no Modified electric convulsive treatment and psychotherapy; 3) baseline HAMD-17 score $\geq 17$; 4) signed the informed consent form. Exclusion criteria: 1) suffering from severe physical illnesses that may interfere with the study treatment; 2) prior episodes of mania or hypomania; 3) pregnant or lactating women, or planned to become pregnant; 4) currently present with serious risks of suicide; 5) having contraindications to MRI scans.

Collect demographic data and perform MRI scans at baseline. All the MDD patients (HAMD-17 scores $\geq 17$ ) in the study group were first episode, never-medicated and without severe complications. The study group was treated with SSRIs, including fluoxetine, escitalopram, citalopram, sertraline, which were initiated at the lowest dose in the commercially available unit and reached to the effective dose on the third day of treatment. All subjects used a single antidepressant. Patients with insomnia could be treated with benzodiazepines for a short time, but this could not be combined with other antidepressants, antipsychotics, electroshock or other physiotherapy within a 2 week period. The subjects from the study group would be assessed for HAMD-17 scale scores at both baseline and after 2 weeks of treatment by the same follow-up physician. After treatment, the score reduction rate $\leq 20 \%$ was non-responder group ( $n=$ 41 ), and the score reduction rate $\geq 50 \%$ was responder group $(n=38)$.

The study was approved by the Institutional Review Board of the First Hospital of Shanxi Medical University (20091217). All the subjects were informed of relevant matters before enrollment, willing to participate in the research, and signed the informed consent form. 


\section{MRI data acquisition}

Data acquisition was performed using the Siemens Trio 3.0T MRI scanner (Germany). The subjects were provided with a sponge mat positioned to restrict head motion and earplugs to reduce noise. All the subjects were required to close their eyes in a resting position and confirm that they had not fallen asleep during the scan. The stereoscopic three-dimensional $\mathrm{T} 1$ weighted images with whole-brain spatial coverage were acquired in the 3D-FLASH sagittal view. The scanning parameters were specified as follows: repetition time $(\mathrm{TR})=2,300 \mathrm{~ms}$, echo time (TE) $=2.95 \mathrm{~ms}$; inversion time (TI) $=900 \mathrm{~ms}$; flip angle $(\mathrm{FA})=9^{\circ}$; field of view $(\mathrm{FOV})=225 \times 240 \mathrm{~m}$; acquisition matrix $=256 \times 240$; slice thickness $=1.2 \mathrm{~mm}$, slice gap $=0.6 \mathrm{~mm}$; voxel size $=1 \times 1 \times 1 \mathrm{~m}$; the scan was performed in the sagittal view with a total of 160 slices. Scan duration=9 $\min 14 \mathrm{~s}$.

\section{MRI data pre-processing}

All 3D T1 structural MRI data were pre-processed using the VBM8 toolbox (http://dbm.neuro.uni-jena.de/vbm.html) of SPM8 (http://www.fil.ion.ucl.ac.uk/spm). ${ }^{18}$ Firstly, for pre-processing, the origin of all T1 MRI data was manually adjusted to the anterior commissure, and the T1 images were normalized to the standard MNI template; the spatially normalized image was next partitioned into GM, white matter and cerebrospinal fluid; the subsequent GM images were modulated to eliminate volume change caused by inter-individual differences in the brain during the normalization process. Finally, the structural image data after segmentation and modulation were smoothed using a Gaussian kernel at $8 \mathrm{~mm}$ full width half maximum (FWHM) for further statistical analysis.

\section{Statistical analysis}

Clinical data were analyzed using SPSS 20.0 (IBM Corp., Armonk, NY, USA). Age, education level, and HAMD-17 scores were tested using independent sample t-test and gender using a chi-square test. Two-sided tests were performed at a significance level of $\alpha=0.05$.

\section{MRI data}

Statistical analysis was performed with GM volume as measures based on the VBM8 toolbox of SPM8 using an independent sample t-test to compare the differential brain regions between all MDD and NCs. A correction was performed using the Gaussian random field theory (RFT). $\mathrm{P}<0.05$ was considered statistically significant. The brain regions showing GM differences between MDD and NC were used as a mask. The difference of GM volume at baseline was compared between responders and non-responders by independent sample t-test. RFT correction was performed to adjust single voxel value at $\mathrm{p}<0.005$, cluster level $<0.05$, after which $\mathrm{p}<0.05$ was statistically significant. The ROC curve was used to analyze the changes of GM volume in corresponding differential brain regions to distinguish the accuracy of the responder group and non-responder group. The GM volume in the differential brain regions between the responder group and the non-responder group was extracted, and the sex, age, and educational background were used as covariates to analyze the correlation between the GM volume and the HAMD-17 reduction rate after 2 weeks of treatment.

\section{RESULTS}

\section{Demographic and clinical data comparisons}

The age and education level of the MDD group and the NC group were analyzed by independent sample t-test and gender by chi-square test. There was no statistically significant difference in age $(\mathrm{t}=-1.38, \mathrm{p}=0.167)$, gender $\left(\chi^{2}=1.108, \mathrm{p}=0.292\right)$ and education level $(\mathrm{t}=-1.206, \mathrm{p}=0.229)$ between the two groups (Table 1).

The age, education level, and HAMD-17 score of the responders and non-responders were analyzed by an independent sample t-test, and the gender was analyzed by the chi-square test. There were no statistically significant differences in age $(\mathrm{t}=-1.63$, $\mathrm{p}=0.106)$, gender $\left(\chi^{2}=3.65, \mathrm{p}=0.056\right)$, and education level $(\mathrm{t}=$ $1.268, \mathrm{p}=0.202$ ) between the two groups (Table 2 ).

\section{Comparison of gray matter volume between MDD and NC}

The smaller GM volume of the right STG, orbital part of right medial frontal gyrus and orbital part of right inferior frontal gyrus was observed in MDD patients versus NCs (Table 3, Figure 1).

\section{Comparison of gray matter volume between responders and non-responders}

The non-responder group demonstrated a significant volume reduction at the right STG compared with the responders, otherwise there were relatively stable findings in the orbital part of the right medial frontal gyrus and the orbital part of the

Table 1. Demographic and clinical data comparisons between MDD group and NC group

\begin{tabular}{lccc}
\hline \multicolumn{1}{c}{ Variable } & MDD $(\mathrm{N}=159)$ & $\mathrm{NC}(\mathrm{N}=53)$ & $\mathrm{p}$ value \\
\hline Age, years $(\overline{\mathrm{x}} \pm \mathrm{s})$ & $33.71 \pm 10.21$ & $35.64 \pm 8.668$ & $0.167^{*}$ \\
Gender (male/female) & $76 / 83$ & $27 / 25$ & $0.292^{\dagger}$ \\
Education level, years $(\overline{\mathrm{x}} \pm \mathrm{s})$ & $4.46 \pm 1.305$ & $4.68 \pm 1.122$ & $0.229^{*}$ \\
HAMD-17 score $(\overline{\mathrm{x}} \pm \mathrm{s})$ & $23.6 \pm 2.4$ & $\mathrm{NA}$ & - \\
\hline
\end{tabular}

${ }^{*}$ t-test, ${ }^{\dagger} \chi^{2}$ test. Education level: $1=$ illiterate, $2=$ primary school, $3=$ junior high school, $4=$ senior high school, $5=$ junior college, $6=$ undergraduate, $7=$ postgraduate and above. MDD: major depressive disorder, NC: normal control, HAMD-17: 17-item Hamilton Depression Rating Scale 
right inferior frontal gyrus (Table 4, Figure 2)

The reduction of GM volume in the right STG was extracted from the responder group and non-responder group and

Table 2. Demographic and clinical data comparisons between responders and non-responders

\begin{tabular}{lccc}
\hline \multicolumn{1}{c}{ Variable } & Responder & Non-responder & \\
& $(\mathrm{N}=38)$ & $(\mathrm{N}=41)$ & $\mathrm{p}$ value \\
\hline Age, years $(\overline{\mathrm{x}} \pm \mathrm{s})$ & $31.17 \pm 10.45$ & $36.37 \pm 12.56$ & $0.106^{*}$ \\
Gender (male/female) & $28 / 13$ & $18 / 20$ & $0.056^{\dagger}$ \\
Education, year $(\overline{\mathrm{x}} \pm \mathrm{s})$ & $4.547 \pm 1.36$ & $4.13 \pm 1.43$ & $0.106^{*}$ \\
HAMD-17 score $(0$ week) & $21.46 \pm 4.84$ & $20.10 \pm 3.03$ & $0.026^{*}$ \\
$(\overline{\mathrm{x}} \pm \mathrm{s})$ & & & \\
$\begin{array}{l}\text { HAMD-17 score }(2 \text { weeks) } \\
(\overline{\mathrm{x}} \pm \mathrm{s})\end{array}$ & $7.58 \pm 3.75$ & $17.92 \pm 3.37$ & $0.000^{*}$ \\
\hline
\end{tabular}

${ }^{*}$ t-test, ${ }^{\dagger} \chi^{2}$ test. Education level: $1=$ illiterate, $2=$ primary school, $3=$ junior high school, $4=$ senior high school, $5=$ junior college, 6=undergraduate, $7=$ postgraduate and above. HAMD-17: 17-item Hamilton Depression Rating Scale

Table 3. Comparison of gray matter volume between MDD and NC

\begin{tabular}{|c|c|c|c|c|c|c|}
\hline \multirow[t]{2}{*}{ Region } & \multirow{2}{*}{$\begin{array}{l}\text { Voxel } \\
\text { size }\end{array}$} & \multirow{2}{*}{$\begin{array}{l}\text { Left/ } \\
\text { right }\end{array}$} & \multicolumn{3}{|c|}{$\begin{array}{l}\text { Peak MNI } \\
\text { coordinates }\end{array}$} & \multirow{2}{*}{$\begin{array}{c}\text { Peak } \\
\text { point } \\
\text { T value }\end{array}$} \\
\hline & & & $\mathrm{x}$ & $\mathrm{y}$ & $\mathrm{z}$ & \\
\hline $\mathrm{MDD}<\mathrm{NC}$ & & & 48 & -6 & -4.5 & -3.5621 \\
\hline STG & 456 & Right & & & & \\
\hline $\begin{array}{l}\text { Orbital part of inferior } \\
\text { frontal gyrus }\end{array}$ & 450 & Right & & & & \\
\hline $\begin{array}{l}\text { Orbital part of medial } \\
\text { frontal gyrus }\end{array}$ & 649 & Right & & & & \\
\hline
\end{tabular}

MNI: montreal neurological institute, MDD: major depressive disorder, NC: normal control, STG: superior temporal gyrus

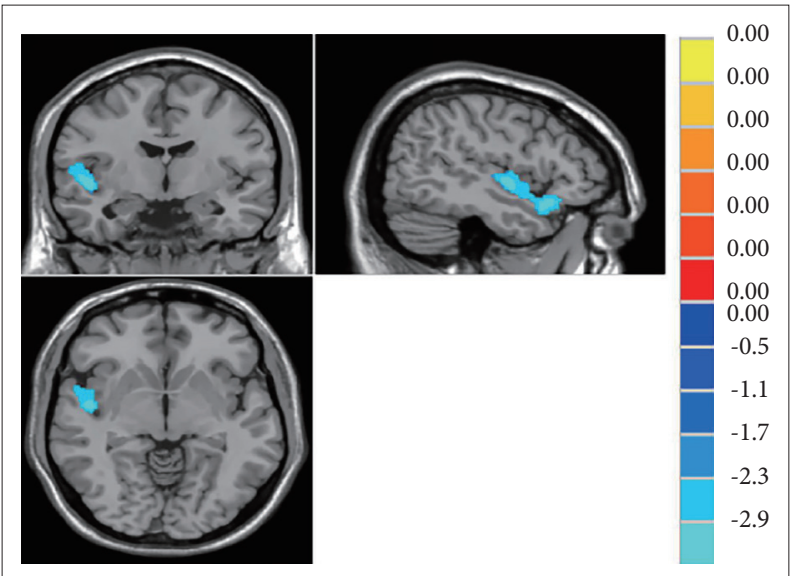

Figure 1. Difference in GM volume between MDD group and NC group. Reductions of GM volume that occurred to the brain were found at the right STG, orbital part of right medial frontal gyrus and orbital part of right inferior frontal gyrus in the MDD group compared with the NC group. GM: gray matter, MDD: major depressive disorder, NC: normal control, STG: superior temporal gyrus. analyzed by the ROC curve. The accuracy of the difference between the responder group and the non-responder group was computed. The curve was drawn with abscissa as 1-specificity and ordinate as sensitivity, and the value of accuray was further calculated. The greater the accuracy value, the higher the accuracy. ROC analysis showed that accuracy $=71.2 \%$. It indicates that it is significant to distinguish the responder group from the non-responder group by the degree of reduction of the GM volume of the right STG (Figure 3).

\section{Relationship between changes in GM volume and drug efficacy in the case group}

The GM volume values of differential brain regions in the responder group and the non-responder group were extracted. Gender, age, and education were taken as covariates, and pearson correlation analysis was conducted with the HAMD17 score reduction rate after 2 weeks of treatment. There was a positive correlation between the STG gray matter volume and the HAMD-17 score reduction rate $(\mathrm{r}=0.347, \mathrm{p}=0.002)$. The larger the GM volume of STG , the better the efficacy of antidepressant treatment. It is suggested that the GM volume of

Table 4. Comparison of gray matter volume between responder and non-responder

\begin{tabular}{ccccccc}
\hline \multirow{2}{*}{ Region } & Voxel Left/ & \multicolumn{2}{c}{ Peak MNI } & Peak \\
\cline { 3 - 4 } & size & right & \multicolumn{2}{c}{$\begin{array}{c}\text { coordinates } \\
\text { point }\end{array}$} & T value \\
\hline
\end{tabular}

Responder $>$ non-responder

STG

$294 \quad$ Right $\quad 55.5 \quad-52.5 \quad 15 \quad 3.4705$

MNI: montreal neurological institute, STG: superior temporal gyrus

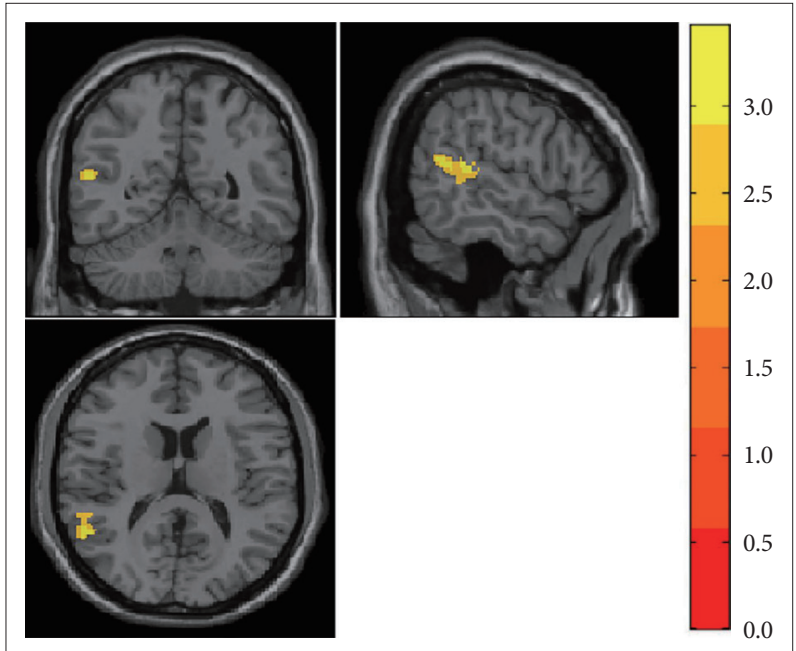

Figure 2. Differences in GM volume between responder group and non-responder group. In the right STG region, all values of responder group-non-responder group were positive (yellow), indicating more significant reductions of gray matter volume in the non-responder group. GM: gray matter, STG: superior temporal gyrus. 


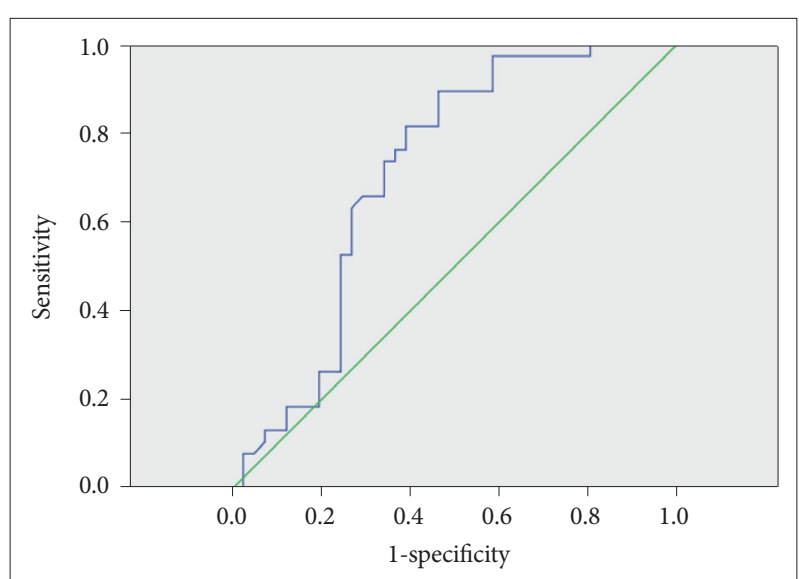

Figure 3. The ROC curve of the degree of reduction of the gray matter of right STG could be used to separate the responder group and the non-responder group. Accuracy $=71.2 \%$. ROC: receiver operator characteristics, STG: superior temporal gyrus.

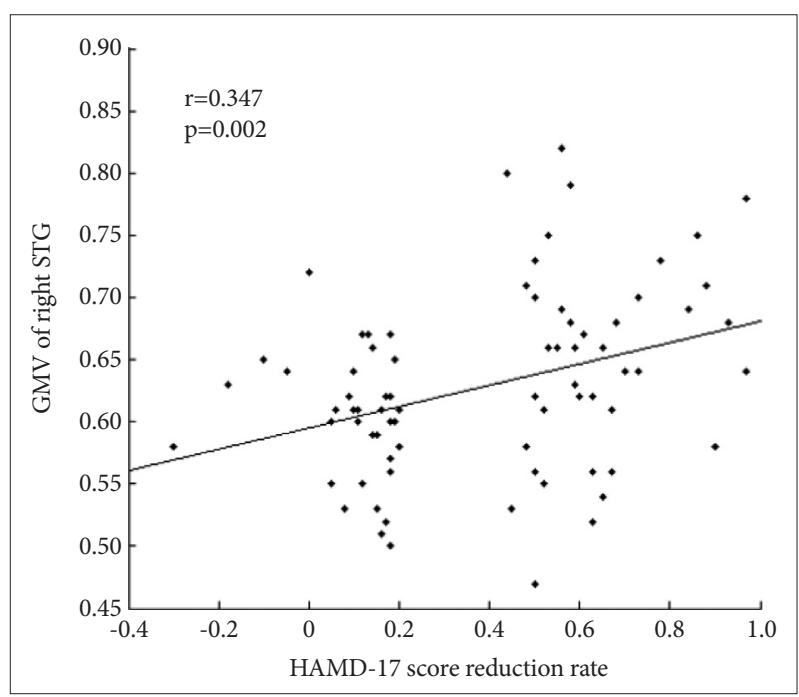

Figure 4. Correlation between the gray matter volume of the right superior temporal gyrus and the HAMD-17 score reduction rate. GMV: gray matter volume, STG: superior temporal gyrus. HAMD17: 17-item Hamilton Depression Rating Scale.

STG may be a biological index to predict the efficacy of drugs (Figure 4).

\section{DISCUSSION}

At present, the pathogenesis and pathophysiological process of MDD are not well understood. Studies of brain morphological structures may help elucidate the effects of this disease on the brain. Depressive disorder is a mood disorder, and studies have shown that ${ }^{19}$ mood disorder is caused by the loss of topdown control of the limbic system. Also, others, including Drevets et al. ${ }^{20}$ and Marchand ${ }^{21}$ introduced the role of functional cortical-subcortical abnormalities, including the limbic-cortical-striatal-pallidal-thalamic circuit as well as medial and orbitofrontal cortex along with their extended cortical circuits.
Magnetic resonance imaging is a non-invasive clinical tool for detecting abnormal brain structures and functions, but the results can be inconsistent and often contradictory. Therefore, further work is needed to understand the importance of these volume changes, ${ }^{22}$ different studies tend to implicate key brain regions to varying degrees. The prefrontal lobe is the associative cortex region of the frontal lobe, which is divided into three main parts: orbital, medial and lateral. The orbitofrontal cortex (OFC) is a subregion of the prefrontal cortex, inferiorly to the superior frontal gyrus (SFG) and the medial frontal gyrus (MFG), and partially at the middle-lower pars triangularis (PT). ${ }^{23}$ OFC controls emotions and affections, mainly collecting the signals transmitted from sensory areas and is highly connected with the limbic brain regions such as the amygdala, which plays a vital role in emotional assessment. This study showed that reductions of gray matter volume observed in the orbital part of the right medial frontal gyrus and right inferior frontal gyrus was the brain structure background for mood and cognitive impairment in MDD patients. The study by Arnone et al. ${ }^{24}$ has shown that unipolar depression is characterized by decreased brain volume that is involved in emotional processing, including the frontal cortex, orbitofrontal cortex, cingulate gyrus, hippocampus, and striatum. The findings of Du et al., ${ }^{3}$ Koolschijn et al. ${ }^{5}$ and Bremner et al. ${ }^{25}$ were consistent with this study, indicating that gray matter reduction in all MDD patients relative to healthy controls was consistently present in the bilateral anterior cingulate cortex (ACC), right medial frontal gyrus and right inferior frontal gyrus, right hippocampus and left thalamus, with gray matter reduction at the right middle-lower frontal gyrus proceeding to the pretemporal region.

Depressive disorder has a significant impact on patients, in addition to tremendous medical and socio-economic burdens. As such, the issue of slow-acting antidepressants along with difficulty in achieving remission urgent needs to be solved. ${ }^{26}$ There is no scientific evidence that any SSRI is more effective than another of this kind, ${ }^{27}$ and the use of the same SSRI drug has not been determined for the pilot study. The SSRIs we used included fluoxetine, paroxetine, sertraline, and escitalopram, which had similar efficacy and tolerability in the treatment of MDD. They were initiated at the lowest dose in the commercially available unit and reached an effective dose on the third day of treatment. During the study, a significant reduction in the volume of right STG was observed through the comparison of differential brain regions between responders and non-responders. Therefore, we preliminarily concluded that the extent to which the volume of right superior temporal gyrus was reduced might be related to its early treatment response. However, not all of the patients responded to the same class of drugs. In the future, we can conduct further comparative studies on different kinds of SSRIs. 
Also, Caetano et al. ${ }^{28}$ and Abe et al. ${ }^{29}$ also reported a reduction in the volume of STG/temporal pole in their studies on depressive disorder. Interestingly, Caetano et al. ${ }^{28}$ founded that the volume of right STG was negatively correlated with the progress of depression. Similarly, the findings by Serra-Blasco et al. ${ }^{30}$ showed that participants with chronic and refractory depression had the smallest volume in the frontotemporal region. Even if this study has not analyzed the volume of specific brain regions yet, the reduction of gray matter volume at the right STG may reflect early treatment response to antidepressants to some extent. The results of this study further demonstrate that the GM volume in the right STG is positively correlated with the early clinical efficacy of depression.

There are still some limitations to this study. At first, our analysis of inter-regional anatomy is based on a correlation analysis of gray matter volume at baseline, as this study is only limited to the first MRI experience. As a result, it is unclear whether the correlation after treatment or prognosis represents a real change in functional connectivity or the parallel effects of pathological physiology of this disease on different brain regions. Further, lacking longitudinal studies does not clarify the findings and clinical significance relevant to the long-term development of the disease.

Overall, this study has found smaller gray matter volume at the right STG, the orbital part of the right medial frontal gyrus and right inferior frontal gyrus by performing structural MRI for a large number of never-medicated patients with firstepisode MDD, thus reconfirming the findings from previous studies of MDD neuroimaging. Since it is not clear whether these parts will have a larger volume after treatment or overtime, a changeable environment, or due to other factors. Sheline et al. ${ }^{31}$ have shown that a longer duration of depressive episodes without antidepressant treatment is associated with decreased hippocampal volume. This indicates that the duration of the disease affects on the change in brain volume. In this study, the duration of the disease has not been studied, and further studies on the correlation between the length of illness and the GM volume in different brain regions can be conducted. In terms of methodology, reasonable longitudinal studies are needed to refine the causal relationship, and to verify whether the reduction of gray matter volume that occurs in specific brain regions predicts the episode of depression and the response to treatment prospectively and whether structural abnormalities are the results of depression. Longitudinal studies can help to describe the factors that predict abnormal development of these brain structures over time. It is also necessary to confirm whether the gray matter volume assessment of these brain regions can be used to identify patients who are likely or unlikely to respond to medications. ${ }^{32}$

\section{Acknowledgments}

This study was supported by the National Key Research and Development Program of China (2016YFC1307103), National Natural Science Youth Fund Project of China (81601192), Shanxi Nature Fund of China (201801D121345). We sincerely thank the patients and their families, as well as healthy volunteers for their participation.

\section{Conflicts of Interest}

The authors have no potential conflicts of interest to disclose.

\section{Author Contributions}

Conceptualization: Yanfang Wang, Qi Meng. Data curation: Qi Meng, Aixia Zhang, YunQiao Zhang. Formal analysis: Qi Meng, Aixia Zhang. Funding acquisition: Yanfang Wang, Ning Sun. Investigation: Xiaohua Cao, Xinrong Li. Methodology: Aixia Zhang, Qi Meng. Supervision: Yanfang Wang. Writing —original draft: Qi Meng. Writing_review \& editing: Aixia Zhang, Xiaohua Cao, Ning Sun, Xinrong Li, YunQiao Zhang.

\section{ORCID iDs}

Qi Meng

Aixia Zhang

Xiaohua Cao

Ning Sun

Xinrong Li

YunQiao Zhang

Yanfang Wang https://orcid.org/0000-0001-5851-1725 https://orcid.org/0000-0002-1335-0005 https://orcid.org/0000-0002-9459-9311 https://orcid.org/0000-0003-3844-2632 https://orcid.org/0000-0003-3266-2116 https://orcid.org/0000-0002-3251-3914 https://orcid.org/0000-0001-9674-3340

\section{REFERENCES}

1. Friedrich MJ. Depression is the leading cause of disability around the world. JAMA 2017;317:1517.

2. Peng W, Chen Z, Yin L, Jia Z, Gong Q. Essential brain structural alterations in major depressive disorder: a voxel-wise meta-analysis on first episode, medication-naive patients. J Affect Disord 2016;199:114-123.

3. Du MY, Wu QZ, Yue Q, Li J, Liao Y, Kuang WH, et al. Voxelwise metaanalysis of gray matter reduction in major depressive disorder. Prog Neuropsychopharmacol Biol Psychiatry 2012;36:11-16.

4. Ashburner J, Friston KJ. Voxel-based morphometry--the methods. Neuroimage 2000;11:805-821.

5. Koolschijn PC, van Haren NE, Lensvelt-Mulders GJ, Hulshoff Pol HE, Kahn RS. Brain volume abnormalities in major depressive disorder: a meta-analysis of magnetic resonance imaging studies. Hum Brain Mapp 2009;30:3719-3735.

6. Videbech P, Ravnkilde B. Hippocampal volume and depression: a meta-analysis of MRI studies. Am J Psychiatry 2004;161:1957-1966.

7. Price JL, Drevets WC. Neurocircuitry of mood disorders. Neuropsychopharmacology 2010;35:192-216.

8. Mayberg HS. Modulating dysfunctional limbic-cortical circuits in depression: towards development of brain-based algorithms for diagnosis and optimised treatment. Br Med Bull 2003;65:193-207.

9. Lai CH. Promising neuroimaging biomarkers in depression. Psychiatry Investig 2019;16:662-670.

10. Guo W, Liu F, Zhang Z, Liu J, Yu M, Zhang J, et al. Unidirectionally affected causal connectivity of cortico-limbic-cerebellar circuit by structural deficits in drug-naive major depressive disorder. J Affect Disord 2015;172:410-416.

11. Guo W, Liu F, Yu M, Zhang J, Zhang Z, Liu J, et al. Functional and anatomical brain deficits in drug-naive major depressive disorder. Prog Neuropsychopharmacol Biol Psychiatry 2014;54:1-6.

12. Yuan H, Zhu X, Luo Q, Halim A, Halim M, Yao H, et al. Early symptom non-improvement and aggravation are associated with the treatment response to SSRIs in MDD: a real-world study. Neuropsychiatr Dis Treat 2019;15:957-966.

13. Tylee A, Walters P. Onset of action of antidepressants. BMJ 2007;334: 
911-912.

14. Malone DC. A budget-impact and cost-effectiveness model for secondline treatment of major depression. J Manag Care Pharm 2007;13(6 Suppl A):S8-S18.

15. Druss BG, Rosenheck RA, Sledge WH. Health and disability costs of depressive illness in a major U.S. corporation. Am J Psychiatry 2000; 157:1274-1278.

16. Revicki DA, Simon GE, Chan K, Katon W, Heiligenstein J. Depression, health-related quality of life, and medical cost outcomes of receiving recommended levels of antidepressant treatment. J Fam Pract 1998;47: 446-452.

17. Mittal VA, Walker EF. Diagnostic and statistical manual of mental disorders. Psychiatry Res 2011;189:158-159.

18. Schaefer A, Burmann I, Regenthal R, Arélin K, Barth C, Pampel A, et al. Serotonergic modulation of intrinsic functional connectivity. Curr Biol 2014;24:2314-2318.

19. Phillips ML, Drevets WC, Rauch SL, Lane R. Neurobiology of emotion perception II: implications for major psychiatric disorders. Biol Psychiatry 2003;54:515-528.

20. Drevets WC, Price JL, Furey ML. Brain structural and functional abnormalities in mood disorders: implications for neurocircuitry models of depression. Brain Struct Funct 2008;213:93-118.

21. Marchand WR. Cortico-basal ganglia circuitry: a review of key research and implications for functional connectivity studies of mood and anxiety disorders. Brain Struct Funct 2010;215:73-96.

22. Cai Y, Liu J, Zhang L, Liao M, Zhang Y, Wang L, et al. Grey matter volume abnormalities in patients with bipolar I depressive disorder and unipolar depressive disorder: a voxel-based morphometry study. Neurosci Bull 2015;31:4-12.

23. Burks JD, Conner AK, Bonney PA, Glenn CA, Baker CM, Boettcher $\mathrm{LB}$, et al. Anatomy and white matter connections of the orbitofrontal gyrus. J Neurosurg 2018;128:1865-1872.

24. Arnone D, McIntosh AM, Ebmeier KP, Munafò MR, Anderson IM. Magnetic resonance imaging studies in unipolar depression: systematic review and meta-regression analyses. Eur Neuropsychopharmacol 2012;22:1-16.

25. Bremner JD, Vythilingam M, Vermetten E, Nazeer A, Adil J, Khan S, et al. Reduced volume of orbitofrontal cortex in major depression. Biol Psychiatry 2002;51:273-279.

26. Portella MJ, de Diego-Adeliño J, Ballesteros J, Puigdemont D, Oller S, Santos B, et al. Can we really accelerate and enhance the selective serotonin reuptake inhibitor antidepressant effect? A randomized clinical trial and a meta-analysis of pindolol in nonresistant depression. J Clin Psychiatry 2011;72:962-969.

27. Gourion D, Perrin E, Quintin P. Fluoxetine: an update of its use in major depressive disorder in adults. Encephale 2004;30:392-399.

28. Caetano SC, Hatch JP, Brambilla P, Sassi RB, Nicoletti M, Mallinger AG, et al. Anatomical MRI study of hippocampus and amygdala in patients with current and remitted major depression. Psychiatry Res 2004;132: 141-147.

29. Abe O, Yamasue H, Kasai K, Yamada H, Aoki S, Inoue H, et al. Voxelbased analyses of gray/white matter volume and diffusion tensor data in major depression. Psychiatry Res 2010;181:64-70.

30. Serra-Blasco M, Portella MJ, Gómez-Ansón B, de Diego-Adeliño J, Vives-Gilabert Y, Puigdemont D, et al. Effects of illness duration and treatment resistance on grey matter abnormalities in major depression. Br J Psychiatry 2013;202:434-440.

31. Sheline YI, Gado MH, Kraemer HC. Untreated depression and hippocampal volume loss. Am J Psychiatry 2003;160:1516-1518.

32. MacQueen GM. Magnetic resonance imaging and prediction of outcome in patients with major depressive disorder. J Psychiatry Neurosci 2009;34:343-349. 